\title{
Shifting Gears in Hippocampus: Temporal Dissociation between Familiarity and Novelty Signatures in a Single Event
}

\author{
Aya Ben-Yakov, Mica Rubinson, and Yadin Dudai \\ Department of Neurobiology, Weizmann Institute of Science, Rehovot 76100, Israel
}

The hippocampus is known to be involved in encoding and retrieval of episodes. However, real-life experiences are expected to involve both encoding and retrieval, and it is unclear how the human hippocampus subserves both functions in the course of a single event. We presented participants with brief movie clips multiple times and examined the effect of familiarity on the hippocampal response at event onset versus event offset. Increased familiarity resulted in a decreased offset response, indicating that the offset response is a noveltyrelated signature. The magnitude of this offset response was correlated, across hippocampal voxels, with an independent measure of successful encoding, based on nonrepeated clips. This suggests that the attenuated offset response to familiar clips reflects reduced encoding. In addition, the posterior hippocampus exhibited an increased onset response to familiar events, switching from an online familiarity signal to an offline novelty signal during a single event. Moreover, participants with stronger memory exhibited increased reactivation of online activity during familiar events, in line with a retrieval signature. Our results reveal a spatiotemporal dissociation between novelty/encoding and familiarity/retrieval signatures, assumed to reflect different computational modes, in response to the same stimulus.

Key words: encoding; episodic memory; fMRI; hippocampus; retrieval

\section{Introduction}

Ample evidence points to hippocampal involvement in human episodic memory (Scoville and Milner, 1957; Squire and ZolaMorgan, 1991; Eichenbaum et al., 1992). The hippocampus has been implicated in all stages of episodic memory, from encoding (Davachi and Wagner, 2002; Paller and Wagner, 2002) through consolidation (Nadel and Moscovitch, 1997; Dudai, 2004) to retrieval (Diana et al., 2007; Wixted and Squire, 2011; Rugg et al., 2012). Neuroimaging studies reveal a spatial dissociation wherein anterior hippocampus is preferentially involved in encoding and posterior hippocampus in retrieval (Lepage et al., 1998; Strange et al., 1999; Ludowig et al., 2008; Spaniol et al., 2009; Poppenk et al., 2013; but see Schacter and Wagner, 1999). Other studies suggest that pattern separation, linked to encoding, occurs primarily in CA3/dentate gyrus, whereas pattern completion, linked to retrieval, occurs primarily in CA1/subiculum (Eldridge et al., 2005; Bakker et al., 2008; Yassa and Stark, 2011). Temporal dissociations have also been suggested, with the hippocampus switching between encoding/retrieval processing modes at various time-

Received May 11, 2014; revised July 8, 2014; accepted Aug. 2, 2014.

Author contributions: A.B.-Y., M.R., and Y.D. designed research; A.B.-Y. and M.R. performed research; A.B.-Y. analyzed data; A.B.-Y. and Y.D. wrote the paper.

This work was supported by the Israeli Center of Research Excellence in the Cognitive Sciences of the Planning and Grants Committee and Israeli Science Foundation Grant 51/11 and by the EP7 Human Brain Project. We are grateful to Micah Edelson, Alex Pine, Avi Mendelsohn, Noga Cohen, and Hagar Goldberg for valuable comments. We also thank Edna Furman-Haran, Fanny Attar, and Nachum Stern from the Norman and Helen Asher Center for Brain Imaging at the Weizmann Institute for technical support.

The authors declare no competing financial interests.

Correspondence should be addressed to Aya Ben-Yakov at the above address. E-mail: aya.benyakov@weizmann.ac.il.

DOI:10.1523/JNEUROSCI.1892-14.2014

Copyright $(C) 2014$ the authors $\quad 0270-6474 / 14 / 3412973-09 \$ 15.00 / 0$ scales (Douchamps et al., 2013), from within theta cycles (Hasselmo et al., 2002; Hasselmo and Stern, 2014) to timescales of seconds (Meeter et al., 2004; Duncan et al., 2012).

Encoding and retrieval are commonly studied using separate experimental designs, yet in reality, these stages are often intertwined, and exposure to familiar stimuli may induce both retrieval and encoding. Studies examining hippocampal processing of novel versus familiar stimuli have led to contradicting conclusions (Kim, 2013). Although most studies that find the hippocampus responds preferentially to novel stimuli (Kirchhoff et al., 2000; Daselaar et al., 2006; Johnson et al., 2008; Kumaran and Maguire, 2009; Suzuki et al., 2011a,b; Kim, 2013; Vannini et al., 2013), others demonstrate the opposite (Montaldi et al., 2006; Kirwan et al., 2009; Reas and Brewer, 2013). This apparent discrepancy may result from masking of familiarity-related signals by novelty-related signals in overlapping hippocampal regions (Yassa and Stark, 2008; Kim, 2013). Notably, Poppenk et al. (2010) found both signals, with encoding of novel stimuli in anterior hippocampus and familiar stimuli in posterior hippocampus.

We demonstrated recently that stimuli that unfold over time (specifically, narrative movie clips) elicit hippocampal encodingrelated activity at the offset rather than the duration of the stimulus (Ben-Yakov and Dudai, 2011; Ben-Yakov et al., 2013). This suggests that hippocampal encoding of complex episodes may not be incremental but concentrated at event offset. Conversely, familiarity-related activity may be expected to occur at stimulus onset, at least for episodes that elicit instantaneous recognition. Thus, we hypothesized that presenting such stimuli multiple times would enable a distinction between encoding-related activity at stimulus offset and familiarity-related activity, expected to occur at stimulus onset. By presenting participants with familiar 
clips in a functional magnetic resonance imaging (fMRI) scanner, we were able to tease apart encoding-related activity and familiarity-induced activity elicited in response to the same events. Increasingly familiar clips yielded signal attenuation in both anterior and posterior hippocampus at event offset. In posterior hippocampus, there was a cooccurring activity increase at event onset. Within posterior hippocampus, novelty and familiarity responses are temporally dissociated, indicating a spatiotemporal dissociation between encoding and retrieval of familiar stimuli.

\section{Materials and Methods}

Participants. Twenty-one right-handed native Hebrew speakers participated in the experiment. One participant was excluded because of a technical error during the experiment, leaving 20 participants in the analysis (14 males, aged $28.9 \pm 1.2$ years). The experimental protocol was approved by the Institutional Review Board of the Sourasky Medical Center (Tel Aviv, Israel), and informed consent was obtained before participation.

Stimuli. The stimuli consisted of 80 brief narrative audiovisual movie clips, in Hebrew (8 s long, dimension $\left.7.5^{\circ} \times 5.7^{\circ}\right)$, presented on a gray background, previously unseen by the participants (Ben-Yakov and Dudai, 2011).

Experimental protocol. The experiment comprised two study phases (Fig. 1) that took place in an fMRI scanner (divided into four runs on day 1 and three runs on day 2) and a test phase (on day 2, 20 min after study). During study phases (Fig. 1a), participants were presented with the movie clips interleaved with fixation screens of variable duration (a red cross on a gray background, $8-16 \mathrm{~s}$ with mean duration of $12.75 \mathrm{~s}$ ). The clips were divided (randomly for each participant) into Repeated (presented six times across study sessions) and Single-Pres (presented once). On day 1, all Repeated clips were presented four times, across the four runs, such that each clip was presented twice during the first two runs (in random order) and twice during the following two runs (in random order). On day 2 , the first run served as a reminder run, in which all Repeated clips were presented (fifth presentation). During the following two runs, all clips (Single-Pres and presentation 6 of Repeated) were presented in random order. Thus, in the first two runs of day 1 , half the clips were novel (Pres1) and half were weakly familiar (Pres2), and in the last two runs of day 2, half the clips were novel (Single-Pres) and half strongly familiar (Pres6). The purpose of this design was to control for any contextual effects. In the additional runs (runs 3-4 on day 1 and the first run of day 2), all the clips were familiar (Pres3-5). The average interval between repetitions was 10.3, 21.3, and $10.4 \mathrm{~min}$ (between presentations $1-2,2-3$, and 3-4, respectively), $1 \mathrm{~d}$ between presentations $4-5$, and $20.5 \mathrm{~min}$ between presentations $5-6$. An $8 \mathrm{~s}$ audiovisual sequence was presented at the beginning of each run to allow for accommodation to the fMRI environment. Additionally, participants were presented with a letter task (to indicate whether a scrambled sentence contained one or more of a class of Hebrew letters that appear at ends of words) twice during each run. Data from this task were not analyzed because its sole purpose was to maintain alertness. All stimuli were presented (Presentation software version 14.9; Neurobehavioral Systems) on a screen behind the participants' head and viewed through a mirror; the corresponding audio was presented using MRI-compatible headphones (MR Confon). Participants were instructed beforehand to view the clips attentively (without engaging in active rehearsal between clips)
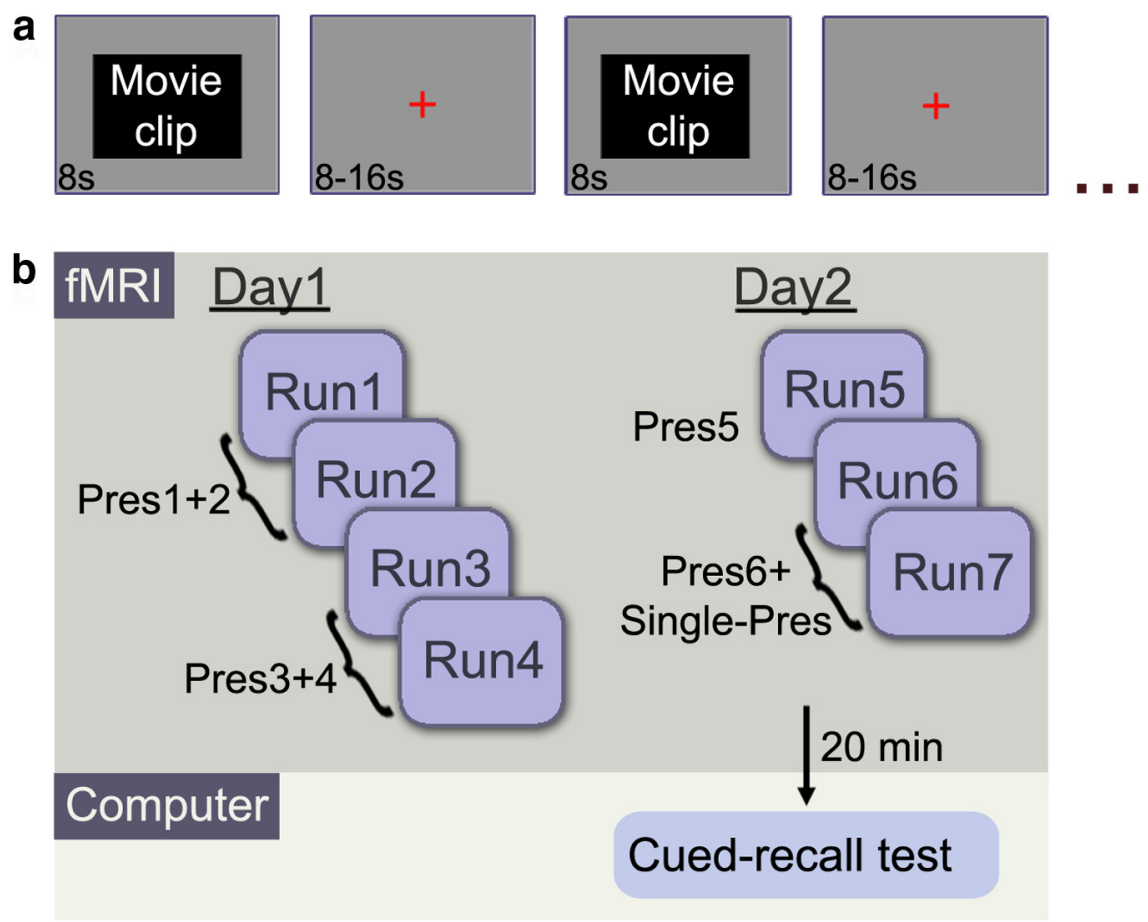

Figure 1. The experimental protocol. $\boldsymbol{a}$, Structure of a single study run. Within each run, 408 -s clips were presented, interleaved with a fixation screen of jittered length $(8-16 \mathrm{~s}$, with a mean duration of $12.75 \mathrm{~s})$. $\boldsymbol{b}$, The study took place over 2 consecutive days, during which 40 clips were presented six times (Pres1-6) and 40 clips were presented once (Single-Pres, only on day 2). The test phase took place 20 min after the last study run on day 2 .

and informed that there would be a subsequent test on the gist of the clips.

The test phase took place outside the scanner, $20 \mathrm{~min}$ after the last study run. It consisted of a self-paced computerized cued-recall test, with one question regarding each clip (in random order, including both Repeated and Single-Pres). The questions were open ended and pertained to the gist of the clips, requiring integration of information over the course of the clip (e.g." "What did the boy say to the girl?"). Each question was accompanied by a frame from the corresponding clip to serve as a visual cue, and participants were instructed to type in their answers in the allotted space or indicate they did not recall the answer by leaving an empty space. After each question that was answered, participants were presented with a visual analog scale (ranging from 0 to 100) and requested to rate their degree of confidence in the preceding answer. Clips corresponding to correctly answered questions were categorized as Remembered, and clips corresponding to unanswered/incorrectly answered questions were categorized as Forgotten. Vague answers, which could not easily be categorized as correct/incorrect, were removed from analysis.

fMRI acquisition and processing. The imaging was performed on a $3 \mathrm{~T}$ Trio Magnetom Siemens scanner at the Ascher Imaging Center, Weizmann Institute of Science, on 2 consecutive days. On each day, there were 4 (day 1 ) or 3 (day 2) functional scanning sessions, followed by an anatomical scan. The functional images were $\mathrm{T} 2{ }^{*}$ weighted and acquired using a gradient-echo echo-planar imaging sequence [repetition time (TR), $2000 \mathrm{~ms}$; echo time (TE), $35 \mathrm{~ms}$; flip angle, $75^{\circ}$; 29 slices with no gap; at $30^{\circ}$ toward coronal from anterior commissure-posterior commissure (ACPC); slice thickness, $3 \mathrm{~mm} ; 3 \times 3 \times 3 \mathrm{~mm}$ resolution; matrix, $80 \times 80$; field of view, $24 \mathrm{~cm}$ ]. The functional coverage included most of the cerebrum (excluding a small portion of the parietal lobe). Anatomical images were acquired using a T1-weighted magnetizationprepared rapid acquisition gradient echo pulse sequence $(1 \times 1 \times 1 \mathrm{~mm}$ resolution; TR, $2.3 \mathrm{~s}$; TE, $2.98 \mathrm{~ms}$; inversion time, $900 \mathrm{~ms}$; flip angle, $9^{\circ}$ ).

All data were preprocessed and analyzed using BrainVoyager QX 2.4 (Brain Innovation) in combination with in-house code (MATLAB 7.12 R2011a; Mathworks) incorporating the Neuroimaging Informatics 
Technology Initiative toolbox (NIFTI; http://research.baycrest.org/ jimmy/NIFTI/) and NeuroElf (version 0.9c; http://neuroelf.net/). Before preprocessing, the first seven volumes from the beginning of each scan were removed (volumes acquired during the initial audiovisual sequence). Initial preprocessing included slice scan-time correction, motion correction, and high-pass filtering. In addition, all events during which an abrupt motion $(>0.4 \mathrm{~mm}$ between two consecutive time points) coincided with a large deviation in the global brain activity $(>1.5$ SD difference from the mean) were excluded from analysis. Before additional analysis, all functional data were aligned to the anatomical data, and then both were realigned to ACPC space. After the initial preprocessing, probabilistic independent component analysis (Beckmann and Smith, 2004) was run, using the Multivariate Exploratory Linear Optimized Decomposition into Independent Components (MELODIC 3.0) tool of the FMRIB (Functional MRI of the Brain) Software Library (version 5.0; http://fsl.fmrib.ox.ac.uk/fsl/fslwiki/FSL). Components identified as noise (Kelly et al., 2010) were then removed from the data.

Anatomical region of interest definition. Anatomical hippocampal regions of interest (ROIs) were defined for each participant using a semiautomated procedure. Freesurfer (http://surfer.nmr.mgh.harvard.edu/) was used for automatic labeling of the hippocampus (Fischl et al., 2002, 2004). The initial labeling was then projected into each participant's ACPC space and manually corrected. The hippocampal ROIs were further segmented to anterior/posterior (AP) sections, at the first coronal slice in which the uncal apex was visible (Weiss et al., 2005; Poppenk and Moscovitch, 2011). The average time course for each ROI was normalized $(z$-score) and used for calculation of ROI-based general linear model (GLM) analyses.

Group-aligned ROIs. Although most analyses were based on the subject-specific anatomical ROIs, a groupwise analysis across hippocampal voxels required alignment of participants' anatomical data and the delineation of a common ROI. Using the Advanced Normalization Tools (ANTs; http://picsl.upenn.edu/software/ants/), a group template anatomy was created, to which all participants' anatomical and functional data were aligned. Bilateral hippocampal group ROIs were then defined manually based on the group template.

GLM analyses. We estimated the response amplitude in the anatomically defined ROIs based on a two-gamma hemodynamic response function (HRF). The onset response was modeled by defining the clip onset as the event and convolving it with the canonical HRF. The offset response, treating the clip offset as the event, was modeled based on parameters derived from an independent dataset (Ben-Yakov and Dudai, 2011; timeto-peak, $7 \mathrm{~s}$; time-to-undershoot-peak, $14 \mathrm{~s}$ ). The rationale for this was to get an optimal estimate, making the fewest a priori assumptions. Because we knew the expected nature of the offset response, which slightly differs from the canonical response to a singular event, we used the previously estimated HRF. In previous studies, which used only novel events, we did not observe an onset response. Thus, we had no a priori expectation regarding the nature of this response and preferred to model it using the canonical HRF. The onset and offset predictors were combined in a single GLM. Because of their temporal proximity, we orthogonalized the onset predictor relative to the offset predictor to remove the shared variance. Notably, orthogonalization in the opposite direction (offset relative to onset) yielded very similar results, likely because of the low shared variance between the original predictors (average variance inflation factor of 1.64). The resulting $\beta$ weights served as response amplitude estimations for testing the effect of familiarity on onset and offset hippocampal activity. The calculation of the overall effect of familiarity was based on the slope of response amplitude across repetitions, calculated separately for each participant. In the initial tests for the effect of repetition, the canonical HRF was used to model the onset response (rather than estimating the parameters from the data) to ensure the validity of the statistical tests. Because the subsequent groupwise analysis across hippocampal voxels did not entail comparison between conditions, the onset model parameters were derived from the data to increase estimation accuracy (timeto-peak, $6 \mathrm{~s}$; time-to-undershoot-peak, $8 \mathrm{~s}$ ).

Trialwise calculation of onset-offset response correlation. For the trialwise correlation of onset-offset effects, we estimated the onset increase and offset decrease for each event of each participant. The onset increase was defined as the difference between Pres6 and Pres 1 of a repeated clip at time $=8 \mathrm{~s}$ and the offset decrease was defined as the difference between Pres 1 and Pres 6 at time $=16 \mathrm{~s}$. Thus, a positive correlation between these measures would indicate an inverse relationship between onset and offset responses at a single-trial level. The correlation was calculated separately per subject, because a different subset of the clips was designated as Repeated for each participant. The Fisher's-transformed correlation values were then used for a group-level $t$ test.

Pattern similarity and pattern enhancement measures. We defined the multivariate activity pattern of the hippocampus based on the raw activity (after $z$-score) of all hippocampal voxels at given time points (without averaging across events), yielding a spatiotemporal pattern. We first used representational similarity analysis (Kriegeskorte et al., 2008) to estimate the similarity between the spatiotemporal patterns of the first and second clip presentations. The similarity was calculated as the Fisher's $z$-transformed linear correlation between the patterns. Pattern similarity represents the degree to which the activity pattern in one condition (here Pres1) is repeated in another (Pres2) and is thus taken as a measure of reactivation. We defined an additional measure of pattern enhancement to test the degree to which the activity pattern of Pres1 was amplified in Pres2 (incorporating not only the similarity between the patterns but the amplitude of the response). Using the spatiotemporal pattern of the first presentation as a model for the second presentation (in a GLM), the resulting $\beta$ weights served as a measure of pattern enhancement. The onset spatiotemporal pattern was based on time points (in resolution of $\mathrm{TR}=2 \mathrm{~s}) 3-5$, and the offset pattern was based on time points 7-9 (around the onset/offset response peaks at TR $=4(8 \mathrm{~s})$ and TR $=8(16 \mathrm{~s})$ respectively). We included in the analysis all functional voxels for which at least two-thirds of the corresponding anatomical voxels were labeled as hippocampal voxels (defined according to the anatomical ROI). Separate patterns were created for the bilateral anterior hippocampus and bilateral posterior hippocampus. This resulted in $91.2 \pm 17.4$ (mean $\pm \mathrm{SD}$ ) voxels in the anterior pattern and $109.1 \pm 12.1$ voxels in the posterior pattern. For example, if a participant had 40 events $\times 3$ time points per event $\times 100$ voxels in the posterior hippocampal ROIs, the spatiotemporal pattern would be a vector of length $40 \times 3 \times 100=12000$.

To test whether the multivariate effect was driven by a univariate effect, we added two additional analyses. First, univariate enhancement was calculated by averaging over voxels, resulting in a temporal pattern (in the example above, averaging over voxels would result in a temporal pattern of length 120). This temporal pattern was used in a GLM, as described above, and the $\beta$ weight served as an estimate of univariate enhancement. Second, we calculated the multivariate pattern enhancement while factoring out the univariate enhancement as a covariate in the original model. We created a predictor in which the value for each voxel in the pattern was replaced by the average across voxels (for each event $X$ time point). Thus, in the example above, the predictor was the same length as the multivariate predictor, but each value $(40 \times 3$ different values) was repeated 100 times, for each voxel. Both predictors were entered into the same model, and the $\beta$ weight of the multivariate predictor served as an estimate of pattern enhancement.

\section{Results}

\section{Memory performance}

Participants recalled $50 \pm 5.5 \%$ (mean \pm SEM) of the NonRepeated clips and $96.7 \% \pm 1 \%$ of the Repeated clips. The average confidence rating for remembered clips, on a scale of $0-100$, was $80.5 \pm 11.6($ mean $\pm \mathrm{SD})$ for Non-Repeated clips and $91.6 \pm$ 6.4 for Repeated clips. These confidence ratings were not incorporated in the analyses because most forgotten clips (an average of $87 \%$ across participants) were left unanswered, and therefore their confidence was not probed. The average confidence rating for the incorrectly answered clips (Non-Repeated only) was $5.1 \pm$ 13.6 (mean $\pm \mathrm{SD}$ ). The analysis correlating novelty with difference due to memory (DM) effects (see Fig. 4) was based on a subset of participants $(n=15)$ with a minimum of 9 Remembered or Forgotten events (as a threshold of 10 resulted in $n=$ 
12). No DM analysis was run on the Repeated clips, because their recall reached ceiling performance. It is noteworthy that participants were informed beforehand that they would be tested; however, they were instructed to avoid intentional rehearsal (see Materials and Methods).

\section{Familiarity increases onset response and decreases offset response in the hippocampus}

By repeatedly presenting the same clips (Fig. 1), we were able to dissociate the effect of familiarity on onset and offset hippocampal blood oxygen level-dependent (BOLD) activity. In both anterior and posterior hippocampus, defined anatomically, we observed a decrease in the offset response with increased familiarity; in the posterior hippocampus, this was coupled with an increase in the onset response (Fig. 2). To test the significance of these effects, we created a GLM with predictors for both the onset and offset response, orthogonalizing the onset predictor with respect to the offset predictor (orthogonalization in the opposite direction yielded very similar results). A three-way repeated-measures ANOVA testing the effect of number of repetitions (Pres\#) on the onset response in anterior/posterior left/right hippocampus revealed a main effect of Pres\# $\left(F_{(5,95)}=\right.$ 2.86, $p=0.02)$ and a significant interaction between the AP axis and Pres\# $\left(F_{(5,95)}=5.93, p=0.0001\right)$. The posterior hippocampus demonstrated a significant increase in the onset response with repetitions (right, $p=0.005$; left, $p=0.002$ ), whereas the anterior hippocampus did not (right, $p=0.99$; left, $p=0.25$ ). Furthermore, the increase in the posterior hippocampus was significantly greater than the anterior in both right $(p=0.0005)$ and left $(p=0.001)$ hippocampus (Fig. 3a). A three-way ANOVA for the offset response revealed again a main effect of Pres\# $\left(F_{(5,95)}=\right.$ 3.36, $p=0.008)$, whereas the interaction with AP axis did not reach significance $\left(F_{(5,95)}=1.65, p=0.15\right)$. In both anterior and posterior hippocampus, there was a decrease in the offset response with repetition (Fig. $3 b$ ). Notably, there was an increase in the offset response between presentations 4 and 5. This increase may arise from the fact that a day passed between these presentations or from a technical reason (Pres5 was the only condition that was presented during a single run rather than across two runs). To verify that there was no difference between the SinglePres and the first presentation of the Repeated, we ran $t$ tests for all ROIs (anterior/posterior left/right hippocampus) for both the onset and offset responses, and all comparisons proved nonsignificant (lowest $p$ value $=0.22$ ). It is important to note that, although repetition had opposing effects on the onset and offset responses in the posterior hippocampus, these were not mirror effects (Fig. 3), suggesting that the onset increase and offset decrease represent two dissociable effects. To further corroborate this, we calculated, for each participant, the correlation between the onset increase and offset decrease at the expected peaks (TR of 4 and $8 \mathrm{~m}$, respectively) across events. These two measures were not significantly correlated
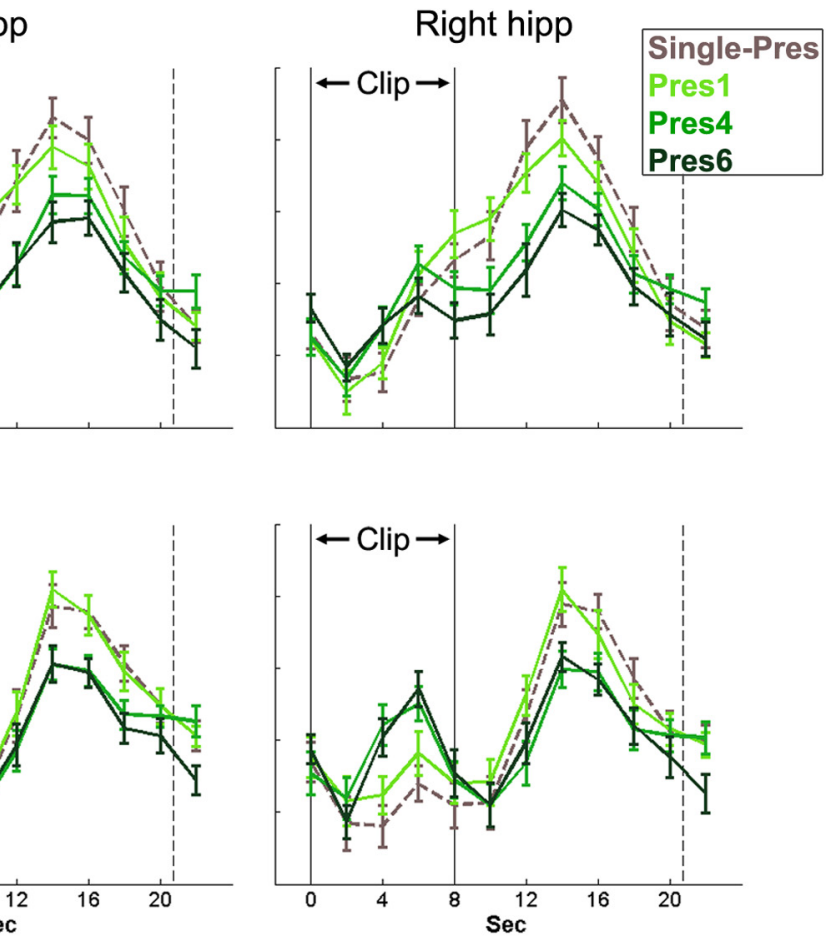

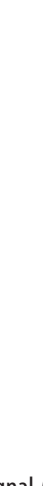

Figure 2. Repetition attenuates offline hippocampal activity and induces an online response. Mean group BOLD signal ( $z$ -
scored) during and after Single-Pres clips and presentations 1, 4, and 6 (Pres1, Pres4, Pres6) of the Repeated clips. The black lines indicate the onset (left line) and offset (right line) of clip presentation, whereas the dashed line indicates the mean onset of the following clip. Error bars represent the SEM.

in either the left (mean $r=0.03, p=0.44$ ) or right (mean $r=$ $-0.02, p=0.7$ ) posterior hippocampal ROIs.

\section{Offset novelty effect correlates with offset DM effect}

We demonstrated previously that hippocampal activity at clip offset is predictive of subsequent memory (Ben-Yakov and Dudai, 2011). To verify the existence of an offset DM effect in the current study, we created a GLM with predictors for the offset of Single-Pres-R, Single-Pres-F (Single-Pres remembered/forgotten) and Pres6 (the Single-Pres clips and the sixth presentation were presented in the same runs, all on day 2). A comparison of Single-Pres-R versus Single-Pres-F (paired $t$ test) in the anatomically defined hippocampal ROIs revealed a significantly stronger offset response to subsequently remembered clips in both left $(p=0.01)$ and right $(p=0.03)$ hippocampus. When dividing to anterior/posterior, there was a significant difference (two-tailed $t$ test $)$ in anterior-left $(p=0.02)$, anterior-right $(p=0.01)$, and posterior-left (0.02) hippocampus. In the posterior-right hippocampus, the difference did not reach significance $(p=0.15)$. Notably, the DM effect was only apparent for offset activity. When testing for a DM effect (one-tailed $t$ test) in either the onset response or the response to the entire duration of clip, no significant difference was observed in any of the ROIs (lowest $p=0.5$ ). Because the offset activity was predictive of successful encoding, the attenuation of this activity with increasing familiarity may reflect reduced encoding. To test this, we correlated the offset novelty effect (difference between Pres1 and Pres6 $\beta$ weights) with the offset DM effect (difference between Single-Pres-R and Single-Pres-F $\beta$ weights) across hippocampal voxels (partial correlation, accounting for general response amplitude by factoring out Pres1, Single-Pres). We found a significant correlation (left/ right hippocampus: partial $r=0.43 / 0.51$, partial $p=5.3 \times 10^{-6} /$ $5.7 \times 10^{-9}$ ) between the novelty effect (i.e., the degree of 


\section{a Onset response:}

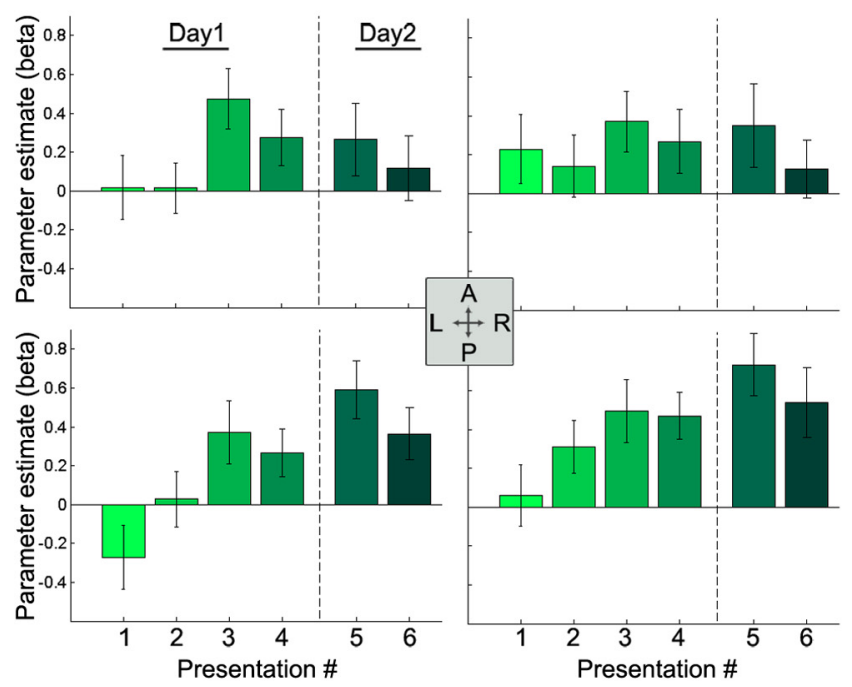

b Offset response:

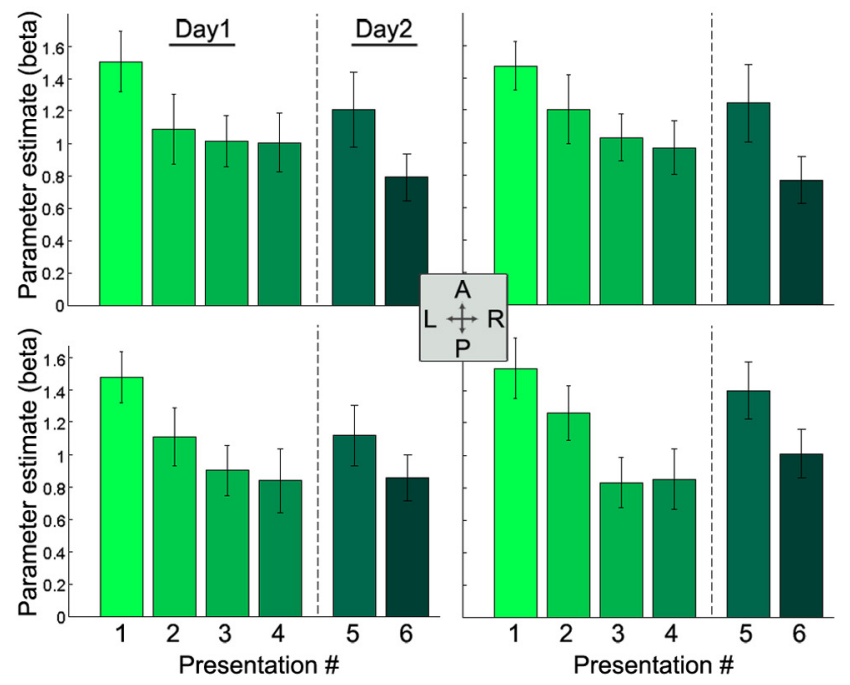

Figure 3. Repeated clip presentation increases online response and decreases offline response in the hippocampus. $\beta$ values for online ( $\boldsymbol{a}$ ) and offline ( $\boldsymbol{b}$ ) responses to presentations $1-6$ of Repeated clips in the anterior (top row) and posterior (bottom row) hippocampus. The posterior hippocampus demonstrates an increased online response with increased familiarity. Both anterior and posterior hippocampus demonstrate a similar decreased offline response. $A$, Anterior; $L$, lateral; $P$, posterior; $R$, rostral.

\section{a Offset response:}

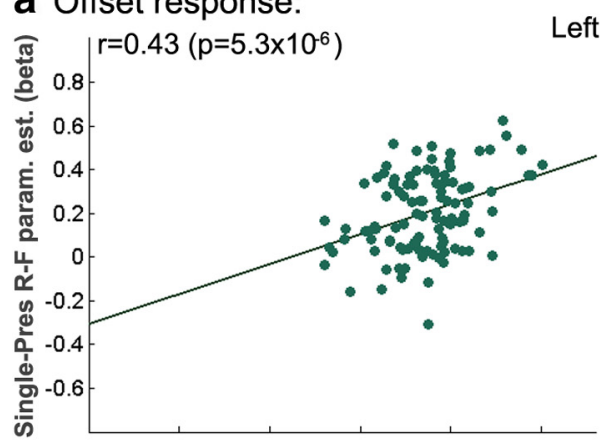

\section{b Onset response:}
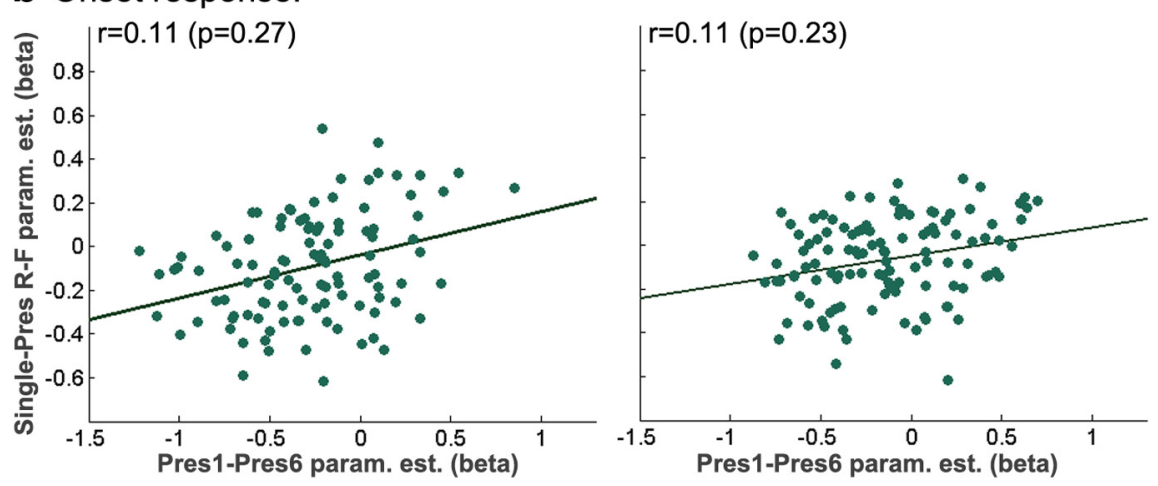

Figure 4. Offline novelty effect correlates with offline DM effect. $\boldsymbol{a}$, The amplitude of the offline novelty effect (difference between the response to Pres 1 and Pres6) plotted against the amplitude of the DM effect (difference between Single-Pres-R and Single-Pres-F). A significant correlation was observed in both right and left hippocampus (after correcting for general response amplitude). $\boldsymbol{b}$, A similar analysis for the online response revealed no significant correlation.

attenuation of brain activation with familiarity) and magnitude of the DM effect (Fig. 4a). Notably, no such correlation was found when correlating the $\beta$ differences for the onset response (Fig. $4 b$ ), and the offset correlation was significantly greater than the onset correlation in both left $(p=0.01)$ and right $(p=$ 0.0007 ) hippocampus, demonstrating additional dissociation between the onset and offset effects.
Onset pattern enhancement correlates with individual memory strength According to influential models, retrieval of memories may entail the reactivation of the medial temporal lobe activity patterns observed during the initial experience (Marr, 1971; Norman and O'Reilly, 2003), a suggestion supported by recent neuroimaging studies (Staresina et al., 2012b; Jafarpour et al., 2013). This raises the possibility that the onset response to familiar clips, primarily in the posterior hippocampus, may reflect reactivation of the activity patterns observed during the initial presentation. We defined onset and offset spatiotemporal activation patterns across hippocampal voxels at the given time points for Pres 1 (first clip presentation) and Pres 2 to separately test for evidence of onset versus offset reactivation. We focused on the similarity between Pres1 and Pres2, because Pres3-6 are likely to incorporate information encoded over multiple previous repetitions. We tested whether the similarity of the Pres 1 and Pres 2 spatiotemporal patterns was linked, across individuals, to memory strength. Memory strength was calculated as the proportion of correctly remembered Single-Pres clips. First, we tested whether the standard reactivation measure (correlation of the two activation patterns; Kriegeskorte et al., 2008) was correlated with the participants' memory performance. We found a significant correlation between onset pattern similarity and performance in the posterior hippocampus (Fig. $5 a, r=0.53, p=0.02$ ), whereas the anterior hippocampus displayed a lower correlation that did not reach significance (Fig. $5 a, r=0.31, p=0.19$, no significant difference in a direct comparison of the anterior and posterior 
a

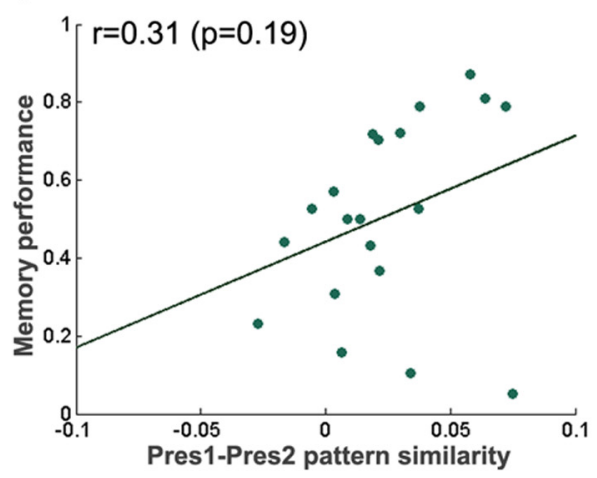

Anterior

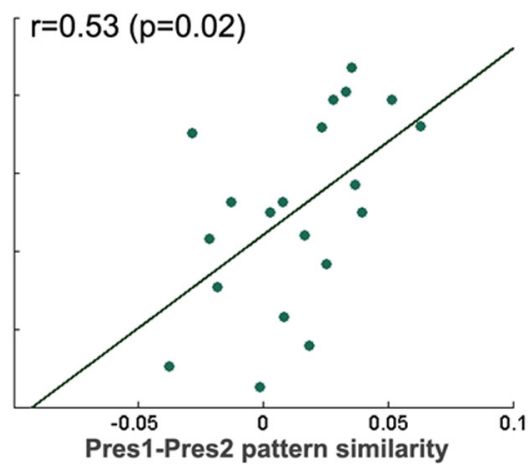

b

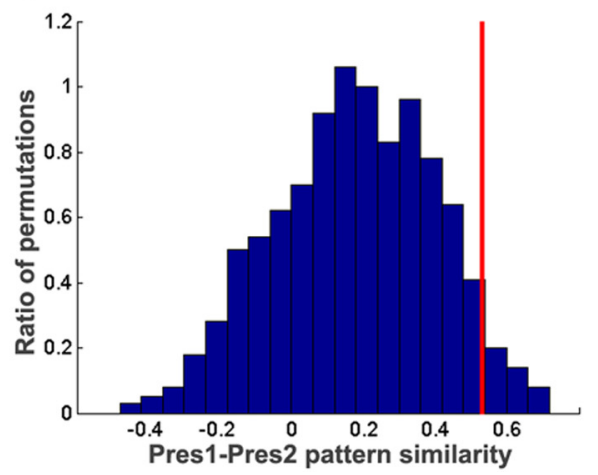

Figure 5. Onset pattern similarity is correlated with individual memory strength. $\boldsymbol{a}$, The similarity (correlation, after Fisher's $z$-correlation) between the online activation pattern in Pres 1 and Pres2 plotted against individual memory strength (determined based on memory for Single-Pres clips). A significant correlation was observed in the posterior hippocampus. $\boldsymbol{b}$, The histogram of Pres1-Pres2 correlations with random permutations of trials (random matching of Pres1 trials to Pres 2 trials). The vertical red line represents the correlation with the correct trial matching $(p=$ 0.048 relative to the random distribution).

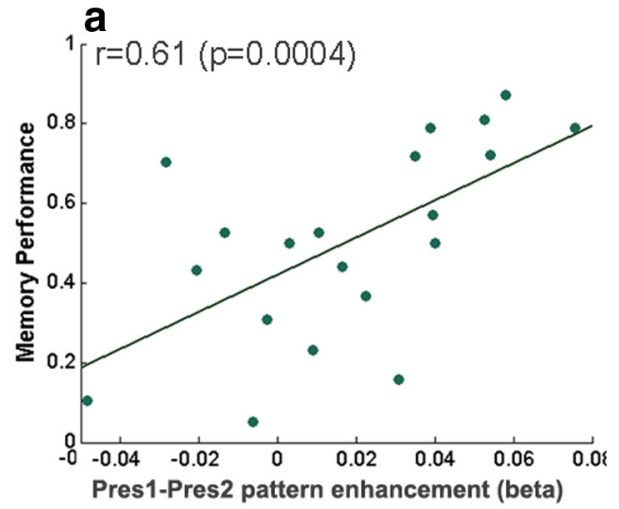

\section{b}

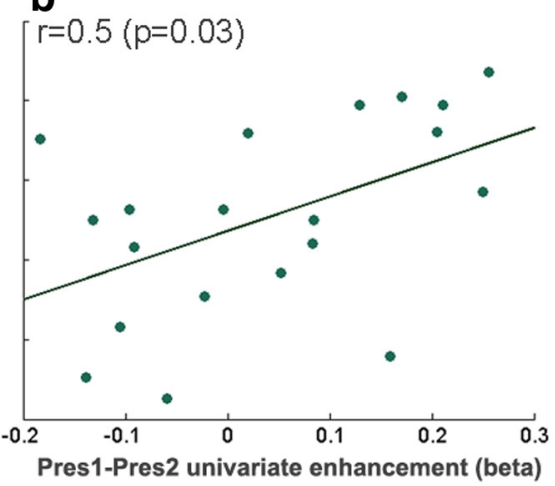

C

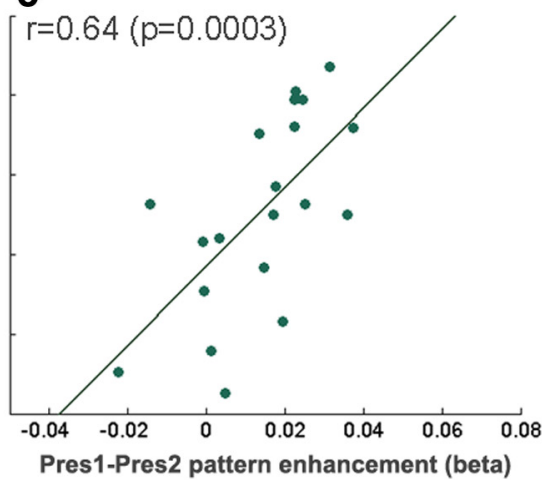

Figure 6. Onset multivariate pattern enhancement is correlated with individual memory strength. $\boldsymbol{a}$, The pattern enhancement between Pres1 and Pres2 (using the spatiotemporal pattern of Pres1 as a model for Pres2) plotted against individual memory strength. $\boldsymbol{b}$, The univariate enhancement between Pres 1 and Pres2 (using temporal patterns, averaged over voxels) plotted against individual memory strength. $\boldsymbol{c}$, Same as $\boldsymbol{a}$ but with the univariate pattern added to the model as a covariate in the calculation of the multivariate pattern enhancement.

hippocampus). When testing the offset pattern similarity, there was no correlation with performance in either anterior $(r=0.15$, $p=0.52)$ or posterior $(r=0.06, p=0.82)$ hippocampus, but the difference between the onset and offset correlation with performance did not reach significance in the posterior hippocampus $(p=0.12)$. Overall, there was evidence of a decrease in similarity to the Pres1 pattern across repetitions in both anterior (mean similarity, $0.024,0.014,0.014,0.007$, and 0.003 ) and posterior hippocampus (mean similarity, 0.013, 0.019, 0.009, 0.008, and 0.007 ), yet the slope of the decrease was significant only in anterior $(p=0.02)$ hippocampus $(p=0.25$ in posterior hippocampus).

To test whether the onset pattern similarity effect reflected trial-specific similarity, we ran a permutation test, with random pairings of Pres1 and Pres2 trials, and for each permutation calculated the correlation of the similarity with performance. In the posterior hippocampus, the similarity for correctly matched trials was significantly higher than the random distribution (Fig. $5 b$, $p=0.048)$, whereas in the anterior hippocampus, there was no significant difference $(p=0.26)$. This suggests that the pattern similarity observed in the posterior hippocampus is event specific.

In addition to the pattern similarity measure, we defined a measure of pattern enhancement incorporating not only the similarity between the patterns but also the amplification of the Pres 1 pattern during Pres2. We used the Pres1 onset pattern as a model for Pres2 (for Repeated clips) and correlated the resulting $\beta$ weights with participants' memory performance on the SinglePres. We found that, in the posterior hippocampus, there was a significant correlation between pattern enhancement and memory performance (Fig. $6 a, r=0.61, p=0.004$ ) that trended to significance even when factoring out the effect of the similarity between the patterns (partial correlation, partial $r=0.42$, partial $p=0.07$ ). This suggests that memory performance is linked not only to the similarity between the patterns but also to the degree of pattern amplification. Thus, the overall increase in the onset response of the posterior hippocampus may reflect enhanced reactivation. In the anterior hippocampus, the correlation was not significant $(r=0.32, p=0.18)$, and the correlation that was observed appeared to arise solely from the similarity (partial $r=$ 0.09 , partial $p=0.72$ ). The correlation of pattern enhancement with performance could arise from either pattern-specific amplification or an overall increase in the hippocampal response magnitude. To test whether the multivariate effect was driven by a univariate effect, we correlated the univariate pattern enhancement with memory performance (Fig. 6b). This revealed a significant, albeit weaker, correlation in the posterior hippocampus $(r=0.5, p=0.03)$, but notably, when controlling for the effect of similarity, the correlation was nonsignificant (partial $r=0.1$, partial $p=0.68$ ). Furthermore, when calculating the multivariate 
pattern enhancement while factoring out the univariate pattern as a covariate, the correlation with behavior was not affected (Fig. $6 c, r=0.64, p=0.0003$, when controlling for similarity partial $r=0.43$, partial $p=0.06$ ).

\section{Discussion}

We presented participants with brief narrative movie clips multiple times and tested the hippocampal response at clip onset and clip offset for effects of stimulus repetition. In addition, we presented participants with nonrepeated clips, each presented once, to obtain an independent measure of encoding-related activation in response to novel events. When encountering novel clips, both the anterior and posterior hippocampus exhibited an immediate offset response, which was predictive of subsequent memory. This offset response was attenuated with repetition. To test whether this attenuation was related to reduced encoding, we correlated the degree of attenuation with the DM effect across hippocampal voxels and found these two effects to be significantly correlated. In parallel, upon encountering familiar clips, an onset response emerged in the posterior hippocampus, exhibiting repetition enhancement. This suggests that the increased online activity in response to familiar events may reflect retrievalrelated processes. Using a multivariate approach, we tested whether this onset response may reflect reactivation of the hippocampal spatiotemporal response pattern at initial encoding, as hippocampal pattern reactivation has been observed previously in repeated retrieval of complex episodes (Chadwick et al., 2010). We found that individuals with superior memory exhibited a higher degree of reactivation in the posterior hippocampus when presented with repeated events.

Across several experiments (Ben-Yakov and Dudai, 2011; Ben-Yakov et al., 2013), we observe a robust hippocampal response that is time-locked to the offset of unfolding stimuli and predictive of subsequent memory. A question arises as to the nature of this offset response, primarily whether it reflects an intentional or automated process (both of which may involve working memory systems; for a more comprehensive discussion of this point, see Ben-Yakov et al., 2013). Although the task was of intentional encoding, accumulating evidence seems to suggest that the offset response does not reflect active rehearsal. First, the response is transient and of a relatively fixed and brief duration. Second, presenting two clips in immediate succession elicits a response at the offset of each clip, and it is not likely that participants are engaged in rehearsing the first clip when encoding the second because there is no proactive interference (Ben-Yakov et al., 2013). In addition, we observe a similar offset DM effect in an incidental task (A.B.-Y. and Y.D., unpublished observations). Drawing from the rodent literature, an intriguing possibility is that the offset response may reflect rapid automated replay (Carr et al., 2011), serving to bind the preceding sequence into a cohesive episode. This binding could potentially be triggered by the occurrence of event boundaries (Kurby and Zacks, 2008). The current study uncovers a key feature of this offline response by demonstrating that it is highly influenced by stimulus novelty. This is especially noteworthy in light of the fact that one of the only other regions originally found to demonstrate an offset DM effect was the striatum (Ben-Yakov and Dudai, 2011), as stimulus novelty is known to modulate the dopaminergic system and its interactions with the hippocampus (Lisman and Grace, 2005; Bunzeck and Düzel, 2006).

Repeated presentation of stimuli is expected to result in hippocampal repetition suppression, presumably because the need for encoding is reduced (Tulving and Kroll, 1995; Tulving et al.,
1996). Indeed, the majority of studies presenting repeated stimuli observe a greater hippocampal response to novel versus familiar stimuli (Kirchhoff et al., 2000; Daselaar et al., 2006; Johnson et al., 2008; Kumaran and Maguire, 2009; Suzuki et al., 2011a,b; Kim, 2013; Vannini et al., 2013; for a meta-analysis, see Kim, 2013), in line with a proposed role of the hippocampus in novelty processing (Kumaran and Maguire, 2009). Specifically, Johnson et al. (2008) reported that, when participants were presented with the same pictures multiple times, both categorical and graded novelty responses were observed in the hippocampus, but no hippocampal regions demonstrated an increase with repetitions (for an additional study demonstrating graded effects, see Vannini et al., 2013). Conversely, from the perspective of retrieval, repeated presentation may be expected to elicit an enhanced response, because the memory trace is likely to become stronger with repetition (Hintzman, 2005). This is supported by studies reporting repetition enhancement in the hippocampus (Montaldi et al., 2006; Kirwan et al., 2009; Greene and Soto, 2012; Reas and Brewer, 2013), as well as studies demonstrating that, among familiar items, there is an increased response to memories that elicit recollection (Daselaar et al., 2006; Montaldi et al., 2006; Suzuki et al., 2011b; Rugg et al., 2012) or a stronger sense of familiarity (Kirwan et al., 2009; Wixted and Squire, 2011).

Several hypotheses have been posited regarding the aforementioned apparent discrepancy. First, both processes may occur in parallel, with the novelty signal canceling out a weaker familiarity signal (Buckner et al., 2001; Stark and Okado, 2003; Kim, 2013), as single-cell recordings have demonstrated both repetition suppression and repetition enhancement within the hippocampus (Viskontas et al., 2006). An alternate, although not mutually exclusive, hypothesis posits that there exists a spatial dissociation such that anterior hippocampus supports novelty/encoding and posterior hippocampus supports retrieval (Lepage et al., 1998; Strange et al., 1999; Spaniol et al., 2009; Poppenk et al., 2013) or encoding of familiar items (Poppenk et al., 2010). Recently, a temporal dissociation has been reported as well, between an early recollection-related response and a late novelty response (suggested to reflect encoding) within the hippocampus (Staresina et al., 2012a). Importantly, the studies discussed above demonstrate dissociation in hippocampal responses to stimuli that elicit a novelty signal and stimuli that elicit a familiarity signal. Here we find both signals in response to the same stimulus. We observe a temporally dissociated increase and decrease in the hippocampal response to the same stimulus, within the same region (posterior hippocampus).

As noted above, novelty signals in the hippocampus have been suggested to reflect increased encoding (Tulving and Kroll, 1995; Brozinsky et al., 2005; Kim, 2013). This is supported by a study that found an overlap between regions demonstrating a novelty response and regions demonstrating a DM effect, including an overlap within the hippocampus (Kirchhoff et al., 2000). We extend this finding by demonstrating a spatial correlation, within the hippocampus, between the magnitude of the DM effect for nonrepeated events and the repetition-induced decrease in the offline response and no correlation in the online response. Whereas a signal that decreases with repetition may reflect encoding processes, an increase across repetitions may reflect retrieval-related processes (Yassa and Stark, 2008). Thus, the signal increase we observe with repetition may reflect retrieval, because there is an increased response to familiar events. This is supported by the finding that the degree to which the activation pattern of the first presentation was reactivated during the second presentation was correlated with individual memory ability. 
Memory ability was correlated not only with pattern similarity but specifically with pattern enhancement. It is tempting to speculate that the increased global onset response to familiar events arises from enhanced reactivation of the activity pattern at encoding. However, we cannot conclusively address this hypothesis here because addition of a recognition task during the study phase in our paradigm would have induced confounding processes of meta-memory, potentially diverting participants' attention from the events themselves to judgment of feeling of knowing.

Intuitively, novelty and familiarity may be considered opposites on a single continuum, with stimulus repetition resulting in decreased novelty and increased familiarity, yet evidence has accumulated that the two are subserved by different brain processes (Daselaar et al., 2006; Kafkas and Montaldi, 2014). Although these studies refer to subjective reports of novelty and familiarity and we examine an objective measure of number of repetitions, we observed a similar dissociation within the hippocampus. During stimulus presentation, we observe a familiarity signal that may reflect retrieval, and at stimulus offset, we observe a novelty response that may reflect encoding. Use of narrative movie clips as naturalistic memoranda, combined with a rest period between each brief clip, enabled us to identify a within-stimulus spatiotemporal dissociation between these two signals, despite the opposing effects of increased familiarity. This paves the way to better understanding of hippocampal involvement in real-life episodic memory, as well as the manner in which the hippocampus can switch between postulated computational modes on demand (e.g., between pattern separation and pattern completion in encoding and retrieval, respectively; Marr, 1971; Norman and O'Reilly, 2003). The multiple types of hippocampal response to a single stimulus can also cast light on mechanisms of reconsolidation and updating of memory and is relevant to issues of memory distortion, in which it is imperative to tease apart the retrieval of the original trace and the encoding of the new, modified trace.

\section{Notes}

Supplemental material for this article is available at http://www. weizmann.ac.il/neurobiology/labs/dudai/uploads/files/Ben_Yakov_ et_al_Supplementary.pdf. The supplemental material includes the following: (1) variations of the analysis in Figure 3 using different measures of estimating the response amplitude; (2) depiction of the estimated HRFs versus the canonical HRF; and (3) more comprehensive discussion of the increase in Pres5 offset. This material has not been peer reviewed.

\section{References}

Bakker A, Kirwan CB, Miller M, Stark CEL (2008) Pattern separation in the human hippocampal CA3 and dentate gyrus. Science 319:1640-1642. CrossRef Medline

Beckmann CF, Smith SM (2004) Probabilistic independent component analysis for functional magnetic resonance imaging. IEEE Trans Med Imaging 23:137-152. CrossRef Medline

Ben-Yakov A, Dudai Y (2011) Constructing realistic engrams: poststimulus activity of hippocampus and dorsal striatum predicts subsequent episodic memory. J Neurosci 31:9032-9042. CrossRef Medline

Ben-Yakov A, Eshel N, Dudai Y (2013) Hippocampal immediate poststimulus activity in the encoding of consecutive naturalistic episodes. J Exp Psychol Gen 142:1255-1263. CrossRef Medline

Brozinsky CJ, Yonelinas AP, Kroll NEA, Ranganath C (2005) Lag-sensitive repetition suppression effects in the anterior parahippocampal gyrus. Hippocampus 15:557-561. CrossRef Medline

Buckner RL, Wheeler ME, Sheridan MA (2001) Encoding processes during retrieval tasks. J Cogn Neurosci 13:406-415. CrossRef Medline

Bunzeck N, Düzel E (2006) Absolute coding of stimulus novelty in the human substantia nigra/VTA. Neuron 51:369-379. CrossRef Medline

Carr MF, Jadhav SP, Frank LM (2011) Hippocampal replay in the awake state: a potential substrate for memory consolidation and retrieval. Nat Neurosci 14:147-153. CrossRef Medline

Chadwick MJ, Hassabis D, Weiskopf N, Maguire EA (2010) Decoding individual episodic memory traces in the human hippocampus. Curr Biol 20:544-547. CrossRef Medline

Daselaar SM, Fleck MS, Cabeza R (2006) Triple dissociation in the medial temporal lobes: recollection, familiarity, and novelty. J Neurophysiol 96: 1902-1911. CrossRef Medline

Davachi L, Wagner AD (2002) Hippocampal contributions to episodic encoding: insights from relational and item-based learning. J Neurophysiol 88:982-990. Medline

Diana RA, Yonelinas AP, Ranganath C (2007) Imaging recollection and familiarity in the medial temporal lobe: a three-component model. Trends Cogn Sci 11:379-386. CrossRef Medline

Douchamps V, Jeewajee A, Blundell P, Burgess N, Lever C (2013) Evidence for encoding versus retrieval scheduling in the hippocampus by theta phase and acetylcholine. J Neurosci 33:8689-8704. CrossRef Medline

Dudai Y (2004) The neurobiology of consolidations, or, how stable is the engram? Annu Rev Psychol 55:51-86. CrossRef Medline

Duncan K, Sadanand A, Davachi L (2012) Memory's penumbra: episodic memory decisions induce lingering mnemonic biases. Science 337:485487. CrossRef Medline

Eichenbaum H, Otto T, Cohen NJ (1992) The hippocampus-what does it do? Behav Neural Biol 57:2-36. CrossRef Medline

Eldridge LL, Engel SA, Zeineh MM, Bookheimer SY, Knowlton BJ (2005) A dissociation of encoding and retrieval processes in the human hippocampus. J Neurosci 25:3280-3286. CrossRef Medline

Fischl B, Salat DH, Busa E, Albert M, Dieterich M, Haselgrove C, van der Kouwe A, Killiany R, Kennedy D, Klaveness S, Montillo A, Makris N, Rosen B, Dale AM (2002) Whole brain segmentation: automated labeling of neuroanatomical structures in the human brain. Neuron 33:341355. CrossRef Medline

Fischl B, Salat DH, van der Kouwe AJ, Makris N, Ségonne F, Quinn BT, Dale AM (2004) Sequence-independent segmentation of magnetic resonance images. Neuroimage 23 [Suppl 1]:S69-S84. CrossRef

Greene CM, Soto D (2012) Neural repetition effects in the medial temporal lobe complex are modulated by previous encoding experience. PLoS One 7:e40870. CrossRef Medline

Hasselmo ME, Stern CE (2014) Theta rhythm and the encoding and retrieval of space and time. Neuroimage 85:656-666. CrossRef Medline

Hasselmo ME, Bodelón C, Wyble BP (2002) A proposed function for hippocampal theta rhythm: separate phases of encoding and retrieval enhance reversal of prior learning. Neural Comput 14:793-817. CrossRef Medline

Hintzman DL (2005) Repetition and memory. In: Psychology of learning and motivation (Bower GH, ed), pp 47-91. New York: Academic.

Jafarpour A, Fuentemilla L, Horner AJ, Penny W, Duzel E (2014) Replay of very early encoding representations during recollection. J Neurosci 34 242-248. CrossRef Medline

Johnson JD, Muftuler LT, Rugg MD (2008) Multiple repetitions reveal functionally and anatomically distinct patterns of hippocampal activity during continuous recognition memory. Hippocampus 18:975-980. CrossRef Medline

Kafkas A, Montaldi D (2014) Two separate, but interacting, neural systems for familiarity and novelty detection: a dual-route mechanism. Hippocampus 24:516-527. CrossRef Medline

Kelly RE Jr, Alexopoulos GS, Wang Z, Gunning FM, Murphy CF, Morimoto SS, Kanellopoulos D, Jia Z, Lim KO, Hoptman MJ (2010) Visual inspection of independent components: defining a procedure for artifact removal from fMRI data. J Neurosci Methods 189:233-245. CrossRef Medline

Kim H (2013) Differential neural activity in the recognition of old versus new events: an activation likelihood estimation meta-analysis. Hum Brain Mapp 34:814-836. CrossRef Medline

Kirchhoff BA, Wagner AD, Maril A, Stern CE (2000) Prefrontal-temporal circuitry for episodic encoding and subsequent memory. J Neurosci 20: 6173-6180. Medline

Kirwan CB, Shrager Y, Squire LR (2009) Medial temporal lobe activity can distinguish between old and new stimuli independently of overt behavioral choice. Proc Natl Acad Sci U S A 106:14617-14621. CrossRef Medline

Kriegeskorte N, Mur M, Bandettini P (2008) Representational similarity 
analysis: connecting the branches of systems neuroscience. Front Syst Neurosci 2:4. CrossRef Medline

Kumaran D, Maguire EA (2009) Novelty signals: a window into hippocampal information processing. Trends Cogn Sci 13:47-54. CrossRef Medline

Kurby CA, Zacks JM (2008) Segmentation in the perception and memory of events. Trends Cogn Sci 12:72-79. CrossRef Medline

Lepage M, Habib R, Tulving E (1998) Hippocampal PET activations of memory encoding and retrieval: the HIPER model. Hippocampus 8:313322. CrossRef Medline

Lisman JE, Grace AA (2005) The hippocampal-VTA loop: controlling the entry of information into long-term memory. Neuron 46:703-713. CrossRef Medline

Ludowig E, Trautner P, Kurthen M, Schaller C, Bien CG, Elger CE, Rosburg T (2008) Intracranially recorded memory-related potentials reveal higher posterior than anterior hippocampal involvement in verbal encoding and retrieval. J Cogn Neurosci 20:841-851. CrossRef Medline

Marr D (1971) Simple memory: a theory for archicortex. Philos Trans R Soc Lond B Biol Sci 262:23-81. CrossRef Medline

Meeter M, Murre JMJ, Talamini LM (2004) Mode shifting between storage and recall based on novelty detection in oscillating hippocampal circuits. Hippocampus 14:722-741. CrossRef Medline

Montaldi D, Spencer TJ, Roberts N, Mayes AR (2006) The neural system that mediates familiarity memory. Hippocampus 16:504-520. CrossRef Medline

Nadel L, Moscovitch M (1997) Memory consolidation, retrograde amnesia and the hippocampal complex. Curr Opin Neurobiol 7:217-227. CrossRef Medline

Norman KA, O’Reilly RC (2003) Modeling hippocampal and neocortical contributions to recognition memory: a complementary-learningsystems approach. Psychol Rev 110:611-646. CrossRef Medline

Paller KA, Wagner AD (2002) Observing the transformation of experience into memory. Trends Cogn Sci 6:93-102. CrossRef Medline

Poppenk J, Moscovitch M (2011) A hippocampal marker of recollection memory ability among healthy young adults: contributions of posterior and anterior segments. Neuron 72:931-937. CrossRef Medline

Poppenk J, McIntosh AR, Craik FIM, Moscovitch M (2010) Past experience modulates the neural mechanisms of episodic memory formation. J Neurosci 30:4707-4716. CrossRef Medline

Poppenk J, Evensmoen HR, Moscovitch M, Nadel L (2013) Long-axis specialization of the human hippocampus. Trends Cogn Sci 17:230-240. CrossRef Medline

Reas ET, Brewer JB (2013) Effortful retrieval reduces hippocampal activity and impairs incidental encoding. Hippocampus 23:367-379. CrossRef Medline

Rugg MD, Vilberg KL, Mattson JT, Yu SS, Johnson JD, Suzuki M (2012) Item memory, context memory and the hippocampus: fMRI evidence. Neuropsychologia 50:3070-3079. CrossRef Medline

Schacter DL, Wagner AD (1999) Medial temporal lobe activations in fMRI and PET studies of episodic encoding and retrieval. Hippocampus 9:7-24. CrossRef Medline
Scoville WB, Milner B (1957) Loss of recent memory after bilateral hippocampal lesions. J Neurol Neurosurg Psychiatry 20:11-21. CrossRef Medline

Spaniol J, Davidson PSR, Kim ASN, Han H, Moscovitch M, Grady CL (2009) Event-related fMRI studies of episodic encoding and retrieval: Metaanalyses using activation likelihood estimation. Neuropsychologia 47: 1765-1779. CrossRef Medline

Squire LR, Zola-Morgan S (1991) The medial temporal lobe memory system. Science 253:1380-1386. CrossRef Medline

Staresina BP, Fell J, Do Lam ATA, Axmacher N, Henson RN (2012a) Memory signals are temporally dissociated in and across human hippocampus and perirhinal cortex. Nat Neurosci 15:1167-1173. CrossRef Medline

Staresina BP, Henson RNA, Kriegeskorte N, Alink A (2012b) Episodic reinstatement in the medial temporal lobe. J Neurosci 32:18150-18156. CrossRef Medline

Stark CEL, Okado Y (2003) Making memories without trying: medial temporal lobe activity associated with incidental memory formation during recognition. J Neurosci 23:6748-6753. Medline

Strange BA, Fletcher PC, Henson RNA, Friston KJ, Dolan RJ (1999) Segregating the functions of human hippocampus. Proc Natl Acad Sci U S A 96:4034-4039. CrossRef Medline

Suzuki M, Johnson JD, Rugg MD (2011a) Decrements in hippocampal activity with item repetition during continuous recognition: an fMRI study. J Cogn Neurosci 23:1522-1532. CrossRef Medline

Suzuki M, Johnson JD, Rugg MD (2011b) Recollection-related hippocampal activity during continuous recognition: a high-resolution fMRI study. Hippocampus 21:575-583. CrossRef Medline

Tulving E, Kroll N (1995) Novelty assessment in the brain and long-term memory encoding. Psychon Bull Rev 2:387-390. CrossRef Medline

Tulving E, Markowitsch HJ, Craik FIM, Habib R, Houle S (1996) Novelty and familiarity activations in PET Studies of memory encoding and retrieval. Cereb Cortex 6:71-79. CrossRef Medline

Vannini P, Hedden T, Sullivan C, Sperling RA (2013) Differential functional response in the posteromedial cortices and hippocampus to stimulus repetition during successful memory encoding. Hum Brain Mapp 34:1568-1578. CrossRef Medline

Viskontas IV, Knowlton BJ, Steinmetz PN, Fried I (2006) Differences in mnemonic processing by neurons in the human hippocampus and parahippocampal regions. J Cogn Neurosci 18:1654-1662. CrossRef Medline

Weiss AP, Dewitt I, Goff D, Ditman T, Heckers S (2005) Anterior and posterior hippocampal volumes in schizophrenia. Schizophr Res 73:103-112. CrossRef Medline

Wixted JT, Squire LR (2011) The medial temporal lobe and the attributes of memory. Trends Cogn Sci 15:210-217. CrossRef Medline

Yassa MA, Stark CEL (2008) Multiple signals of recognition memory in the medial temporal lobe. Hippocampus 18:945-954. CrossRef Medline

Yassa MA, Stark CEL (2011) Pattern separation in the hippocampus. Trends Neurosci 34:515-525. CrossRef Medline 